\title{
ORIGINAL ARTICLE \\ Grand-paternal age and the development of autism-like symptoms in mice progeny
}

\author{
S Sampino ${ }^{1,2}$, GR Juszczak ${ }^{2}$, F Zacchini ${ }^{1}$, AH Swiergiel ${ }^{3,4}$, JA Modlinski ${ }^{2}$, P Loi ${ }^{1}$ and GE Ptak ${ }^{1,2}$
}

Advanced paternal age (APA) contributes to the risk of autism spectrum disorders (ASDs) in children. In this study, we used a mouse model to investigate the effects of APA on behavioral features related to autistic syndromes (that is, social deficits, communication impairments and stereotypic/repetitive behaviors). We also examined whether such effects are transmitted across generations. To do this, males aged 15 months (APA) and 4 months (control) were bred with 4-month-old females, and the resulting offspring (F1) and their progeny (F2; conceived by 4-month-old parents) were tested for the presence and severity of ASD-like behaviors. Our results indicate that APA resulted in offspring that displayed distinctive symptoms of ASD. We found that both F1 conceived from old fathers and F2 derived from old grandfathers displayed increased ultrasound vocalization (USV) activity, decreased sociability, increased grooming activity and increased anxiety-like responses. Moreover, such abnormalities were partially transmitted to the second generation of mice, having APA grandfathers. In conclusion, our study suggests that the risk of ASD could develop over generations, consistent with heritable mutations and/or epigenetic alterations associated with APA.

Translational Psychiatry (2014) 4, e386; doi:10.1038/tp.2014.27; published online 29 April 2014

\section{INTRODUCTION}

Autism spectrum disorders (ASDs) are common and heterogeneous neuropsychiatric conditions characterized by deficits in social interaction, impaired communication, repetitive/stereotypic behavior and/or restricted interests. ${ }^{1}$ ASDs are considered highly heritable neuropsychiatric diseases, suggesting that genetic factors, including de novo mutations, chromosomal abnormalities and common genetic variants, have an important role in its etiology. ${ }^{2-4}$ However, ASD displays considerable genetic heterogeneity, including several regions of the genome which might contribute to its etiology. In addition to genetic variation, several environmental factors have been identified, including obstetric complication, preclampsia, maternal infection and advanced paternal age (APA), which increase the risk of ASD diagnonis. ${ }^{5-7}$ Nevertheless the genetic architecture and the factors influencing the development of ASD are becoming increasingly studied, its neurobiology and pathogenesis, as well as the application of therapeutic and bio-diagnostic tools, are still far to be completely elucidated.

Several epidemiological reports suggest a strong association between APA and an increased risk of conceiving children with ASD. $^{7-11}$ The causal link of this association is suggested to be related to an increased frequency of mutations and/or epigenetic alterations in the germ line of older male individuals. ${ }^{12,13}$ Due to the constant turnover of spermatogonial stem cells, the number of cell replication in the male germline increases with age, increasing the susceptibility to copy errors and resulting in rare de novo mutations affecting individual sperms and then offspring. Recent large-scale sequencing studies have shown that approximately $80 \%$ of de novo point mutations are from paternal origin, and that the rate of mutation increases with age. ${ }^{14-16}$ Therefore, agerelated mutagenesis could represent a triggering factor in the development of ASD and many other psychiatric disorders characterized by a strong genetic contribution.

Mouse models have been used to understand genetic and environmental contributions to ASD, establishing potential etiologies in clinical populations and dissecting the effects of single genes and prenatal factors on specific behavioral features of the mouse. ${ }^{17-25}$ In this context, animal models of APA represent a natural platform to study how mutations and epigenetic errors contribute to the development of ASD, as well as their neurobiology and pathogenesis. Although previous rodent models assessed long-term effects of APA on offspring behavior, the range of behavioral outcome differ substantially among these and it is not known whether APA mice present specific face validity with respect to ASD. ${ }^{26-28}$ In this study we used a mouse model to explore the effects of APA on various behavioral features related to the core symptomatology of ASD. Moreover, we analyzed whether these effects are transmitted to further generations, to verify genetic heritability. Our results support the hypothesis that APA increases the risk of autism in mice and that this risk could develop over generations.

\section{MATERIALS AND METHODS}

\section{Animals}

All animal experiments were performed in accordance with the European Community regulation $86 / 609$. Swiss albino mice originated and maintained at the Institute of Genetic and Animal Breeding (Jastrzebiec, Warsaw, Poland), housed in $30 \times 13 \times 11 \mathrm{~cm}$ cages, under controlled light and temperature, and were given ad libitum access to water and food (Labofeed H, Kcynia, Poland; metabolic energy of $13.0 \mathrm{MJ} \mathrm{kg}^{-1}$ ). To obtain F1 offspring, virgin males aged 15 months (APA; $n=9$ ) and 4 months (control (CTR); $n=10$ ) were bred with mature females, aged 4 months. To obtain the F2 generation, F1 males were bred with unfamiliar females,

\footnotetext{
${ }^{1}$ Faculty of Veterinary Medicine, University of Teramo, Teramo, Italy; ${ }^{2}$ Institute of Genetics and Animal Breeding, Polish Academy of Sciences, Jastrzebiec, Poland; ${ }^{3}$ Faculty of Biology, University of Gdansk, Gdansk, Poland and ${ }^{4}$ Department of Pharmacology, Toxicology and Neuroscience, Louisiana State University Health Sciences Center (LSUHSC), Shreveport, LA, USA. Correspondence: Dr GE Ptak, Faculty of Veterinary Medicine, University of Teramo, p.za aldo moro 45, 64100 Teramo, Italy. 
both aged 4 months. After all pregnancies were detected by the presence of a vaginal plug, males were removed and each dam was singly housed and allowed to deliver spontaneously. If no plug was detected after overnight male-female encountering, the female was removed and replaced by another, to avoid any possible effects of longer contact with the male. The day of delivery was designated as postnatal day 1 (P1). A cohort of animals ( $n=40$ per group) was used to study pre-weaning behaviors. Another cohort ( $n=15$ per group per sex) was weaned on P21, housed in sex-matched groups of $4-5$ in $30 \times 13 \times 11 \mathrm{~cm}$ cages, and subsequently screened for adult behavior. In addition, C57/CBA hybrid mice were used as stimulus mice for the three-chambered social test.

\section{Experimental procedure}

On P2, the skin of each F1 and F2 animal was labeled with a permanent, waterproof pen. Individuals were randomly selected from the litter and assigned to the various analyses. In each generation, a subgroup of mice ( $n=15$ per group per generation) was used to assess developmental milestones on P2-20: body weight, body length, ear/eyes opening, hair appearing, incisive eruption and righting reflex ability. A second subgroup ( $n=25$ per group per generation) was analyzed at P4, 8 and 12 for isolation-induced ultrasound vocalization (USV) and at P10 for homing testing. An independent cohort of male mice was left undisturbed until weaning and used to screen adult behaviors starting at 2.5 month of age ( $n=12-15$ mice per group per generation). Each behavioral test was performed as reported in the Supplementary Table. Because autism is more common in male individuals (by a ratio of 4:1), adult behavior was analyzed only in males. ${ }^{29}$

\section{Body development}

Pups were weighed every two days from P2 to P20, and their body length was measured from the tail tip to the nose. Physical landmarks, including eye/ear opening, incisor eruption and fur development, were scored during the pre-weaning period.

\section{Righting reflex}

This test allows the measurement of the postural reflex in mice at an early age. The mother was separated from the litter $30 \mathrm{~min}$ before testing, and the home cage containing the pups was carried into the test room and maintained at $25^{\circ} \mathrm{C}$ on a heating pad. Each animal was turned on its back (ventral side up) on a flat surface and given a maximum of $60 \mathrm{~s}$ to return to the four paws position. Time of success was recorded for three consecutive trials for each pup. The test was performed on P6 and P10.

\section{Homing test}

This procedure exploits the tendency of immature pups to maintain contact with the mother and siblings. On P10, mother was removed and litter was kept in the home cage at $25^{\circ} \mathrm{C}$ on a heating pad. The floor of a rectangular arena (measuring $36 \times 22 \times 10 \mathrm{~cm}$ ) was subdivided into three areas, one of which was uniformly covered with wood shavings from the home cage, thus containing familiar odor stimuli. The remaining space was covered with clean bedding material. Individual pups were placed on the opposite side of the nest materials, onto a wire mesh located above the arena divided in nine squares, and were allowed to freely move around for $4 \mathrm{~min}$. Total lines crossed, latency time to reach the nest area, time spent in the nest area and the number of entries into the nest area were recorded as an index of homing performance. ${ }^{30}$

\section{Isolation-induced USV}

Testing for isolation-induced vocalization was done on P4, P8 and P12 as described previously, ${ }^{31}$ with slight modifications. Briefly, pups were isolated one by one from their mother and littermates and placed in a clean plastic container into a sound-attenuating chamber, under controlled temperature $\left(28^{\circ} \mathrm{C}\right)$. To record vocalizations, an ultrasound-sensitive microphone-a bat detector (US Mini-2 bat detector, Summit, Birmingham) tuned in the range of $60-80 \mathrm{kHz}$ - was suspended above the isolated pup and USVs were recorded for 5 min using Audacity software (http:// audacity.sourceforge.net/). For analysis, recordings were imported into Avisoft SASLab software (Avisoft Bioacoustics, Glienicke, Germany). Background noise was removed, and call detection was provided by an automatic threshold-based algorithm. An experienced human observer checked the accuracy of call detection, and obtained a $100 \%$ concordance between automated and observational detection. Parameters analyzed included the total number, sound pressure amplitude and duration of calls. No differences in patterns of calling were detected in a comparison of male and female pups, therefore data were collapsed across sex. A subgroup of mice ( $n=15$ /group) in both F1 and F2, was isolated in the presence of nest bedding material to analyze the effects of familiar environmental cues on USV activity. ${ }^{32,33}$

\section{Adult behavioral screening}

In adults, each behavioral test was conducted starting at P75, in the following order: sociability and social novelty (P75), open field (P83), elevated plus maze (P91), observation of repetitive behaviors (P99), tail suspension (P106) and startle reflex (P115). There was at least one week of interval between tests performed on the same animal. The tests were ordered such that the most stressful were performed last. Each apparatus was washed with $70 \%$ ethanol and rinsed with water between subjects to avoid olfactory cueing behaviors. All behavioral tests were made blind to experimental conditions and videotaped for further detailed analysis. Social tests were performed using the EthoVision 3.1 video analysis system (Noldus Information Technology, Wageningen, The Netherlands). All tests were performed during the end of the light phase in an experimental room isolated from the colony. Mice were introduced into the testing room $1 \mathrm{~h}$ before testing to permit environmental habituation, and were returned to their home cage at the end of each test.

\section{Three chambers social tests}

To test sociability and preference for social novelty, adult mice were tested as described previously, ${ }^{34,35}$ with slight modifications. Each mouse was tested in a glass box $(60 \times 40 \times 50 \mathrm{~cm})$ divided by plexiglass walls into three chambers. The test mouse could freely move between chambers through a small opening $(6 \times 6 \mathrm{~cm})$ present in each wall. At the beginning of the test, the test mouse was placed in the central chamber and allowed to freely explore the empty apparatus for $20 \mathrm{~min}$ to evaluate bias for either of the side chamber. After this habituation period, the test mouse was removed from the apparatus and briefly placed in a clean cage. An unfamiliar C57/ CBA 3-month-old male (stranger 1 ) was placed inside a small wire cage in one of the side chambers and an identical empty wire cage was placed in the opposite chamber as a non-social object. Then the test mouse was again placed in the central chamber and allowed to freely explore all three chambers for $10 \mathrm{~min}$. The time spent in each chamber was measured with EthoVision. The preference index for sociability was calculated as (timesocial $\left(\right.$ time $^{\text {social }}+$ time $\left.\left.^{\text {non-social }}\right)\right) .{ }^{7}$ In addition, a human observer scored the time the mouse spent sniffing each wire cage. The location of the stranger 1 alternated between left and right sides of the box across subjects. The animals serving as strangers had previously been habituated to placement in the small cage.

At the end of the sociability test, each mouse was tested in another 10minute session to quantitate its preference to spend time with a newlyintroduced stranger (social novelty) versus an already-investigated mouse. The test mouse again was removed from the apparatus and briefly placed in a clean cage. A new, unfamiliar C57/CBA 3-month-old male was placed in the wire cage that had been empty during the previous 10-minute session. The test mouse was then placed in the central chamber and had a choice between the first, already-investigated, now-familiar mouse (stranger 1) and the novel, unfamiliar mouse (stranger 2). Measurements were taken as described above for the sociability test. ${ }^{18}$

\section{Open field}

Open field was used to assess anxiety-related behavior and general locomotor activity. The apparatus consisted of a square arena $(60 \times 60 \mathrm{~cm})$ divided into 16 squares and enclosed by continuous walls, each $30 \mathrm{~cm}$ in height. The 12 squares adjacent to the wall represented a protected field, whereas the four central squares represented an exposed field, namely 'central area'. The test was initiated by placing a single mouse in the center of the arena and letting it move freely for $6 \mathrm{~min}$. A number of conventional parameters were collected during the session: (i) time to leave the center-the duration of time the mouse spent before leaving the central area; (ii) locomotory activity - the number of lines crossed by the mouse during the entire test; (iii) the number of entries into the central area; and (iv) the cumulative time spent in the central area. ${ }^{36}$ 


\section{Elevated plus maze}

Elevated plus maze was used to assess exploration activity and anxiety-like behavior. The apparatus consisted of two open arms $(30 \times 5 \mathrm{~cm})$ and two closed arms $(30 \times 5 \mathrm{~cm})$, enclosed by $15-\mathrm{cm}$ high walls, with the two pairs of identical arms emerging oppositely from a central platform $(5 \times 5 \mathrm{~cm})$. The apparatus was elevated $45 \mathrm{~cm}$ above the floor. The test was initiated by placing the mouse on the central platform, facing one of the closed arms and letting it move freely for $6 \mathrm{~min}$. A number of conventional parameters were collected during the session: (i) total arms entries-the total number of entries with all four paws into both the open and closed arms; (ii) open arms entries-the number of entries into the open arms with all four paws; (iii) open arms exploration-the number of times the mouse entered the open arms with only its forward paws (stretch-attend posture); and (iv) time spent in the open arms-the cumulative time spent in the open arms. ${ }^{36}$

\section{Assessment of repetitive behaviors}

The mouse was isolated in a new cage filled with clean bedding. After $10 \mathrm{~min}$ of habituation, the animals were video recorded for an additional $10 \mathrm{~min}$ and later scored for self-grooming and digging behaviors by an observer blinded to the experimental groups. Grooming was defined as time spent licking paws, washing nose or face or scratching fur with any foot. ${ }^{37}$ Digging was defined as time spent actively moving the bedding.

\section{Tail suspension}

This test was performed as we described previously. ${ }^{38,39}$ Briefly, a short piece of paper adhesive tape (about $6 \mathrm{~cm}$ ) was attached along half the length of the tail. The free end of the tape was attached to a $30 \mathrm{~cm}$ long rigid tape, which hung from a horizontal bar clamped to a heavy laboratory support stand. Suspended animals were surrounded by a black wooden enclosure $(45 \mathrm{~cm}$ high, $40 \mathrm{~cm}$ wide, $40 \mathrm{~cm}$ deep) such that the mouse's head was $\sim 20 \mathrm{~cm}$ above the floor. For testing, each mouse was suspended by its tail and observed for 6 min. An observer scored the total duration of a passive, 'dead weight' hanging (immobility), between the periods of wriggling of the animal to avoid the aversive situation.

\section{Acoustic startle reflex (ASR) and prepulse inhibition}

The animals were tested for acoustic startle reflex (ASR) with prepulse inhibition with the Startle box (Med. Associates, St. Albans, VT, USA). The mouse was placed in a plexiglass cylinder within a sound-attenuating chamber, upon a piezoelectric transducer, which allowed movements to be quantified and displayed on a computer. An external digital sound level meter (Med. Associates) was used to confirm the calibration of acoustic stimuli before each day of testing. Each test consisted of three blocks of trials, for a total of 40 trials, presented after 5 min of acclimation under $60 \mathrm{~dB}$ of ambient white noise. The first and the last blocks consisted of five trials in which a $120 \mathrm{~dB}$ white noise stimulus was presented alone without prepulse (ASR-simple). The central block consisted of a total of 30 stimuli, presented as $120 \mathrm{~dB}$ white noise signals with or without prepulse inhibition. Prepulses (at 70, 75, 80 and $85 \mathrm{~dB}$ ) in this central block were presented in pseudo-random order $100 \mathrm{~ms}$ before the $120 \mathrm{~dB}$ main stimulus (ASR-pp). Inter-trial intervals varied randomly from 10 to $20 \mathrm{~s}$. The ASR was defined as the movement amplitude after the $120 \mathrm{~dB}$ stimulus, resulting in a peak value. Prepulse inhibition for each prepulse intensity was calculated as a percentage reduction in mean of ASR-pp compared with ASR-simple, according to the formula (ASR-pp/ASR-simple) $\times 100$. Habituation was calculated from the differences in mean response of the first block compared with the last block of ASR-simple stimuli, according to the formula ((ASR block3 - ASR block 1)/ASR block 1) $\times 100-100{ }^{40}$

\section{Statistical analysis}

Data were computed using GraphPad Prism (version 5.0). Each behavioral variable was analyzed in each generation, comparing APA and CTR with one-way analysis of variance (nonparametric Kruskal-Wallis test+Dunn's multiple comparison test). For neonatal tests, data were collapsed across sexes. Differences were considered statistically significant when $P$-values $<0.05$.

\section{RESULTS}

Normal general health, neurological reflexes, homing ability and depression-like behavior

Body weight and length were not affected by APA in both generations analyzed. Analysis of markers of somatic growth did not reveal any changes in body development. Both APA and CTR pups of both generations had completed pinnae detachment by P14; incisor eruption began on P7 and continued until P14; opening of the eyes was completed by P13; and fur started to appear around P10. The righting reflex was not affected by APA, as by $\mathrm{P6}$, the latencies to return to the four paws position were similar among all mice analyzed $(P=0.14)$. On $\mathrm{P} 10$, all pups fully developed the righting reflex, and were able to successfully complete the test within $1 \mathrm{~s}$. No significant differences were found in homing performance. Both the latency to score and the time spent into the area containing nest materials were similar in all pups analyzed. Moreover, no effect of APA was found in the startle reflex and in the time of immobility in the tail suspension test.

\section{Increased USV activity in APA progeny}

The number of calls elicited by social isolation in pups differed across postnatal days $(P<0.0001)$ with more calls observed on P8 and fewer on P12 (Figure 1a). USV emissions by APA pups were much more numerous than those of CTR pups, in both F1 and F2 progeny on P4 and P8 (Figure 1a). Analysis of USV sound amplitude revealed an increased percentage of high intensity calls in APA F1 and F2 progeny on P4 and P8 (Figure 1b). When pups were isolated in the presence of nest material, the rate of calls was attenuated in all mice analyzed. Comparing APA and CTR pups under this particular condition, we did not find any significant difference in USV parameters (Figure 1c). Also, no significant sex differences were observed $(P=0.64)$, nor did we find any differences in motor activity.
USV rate

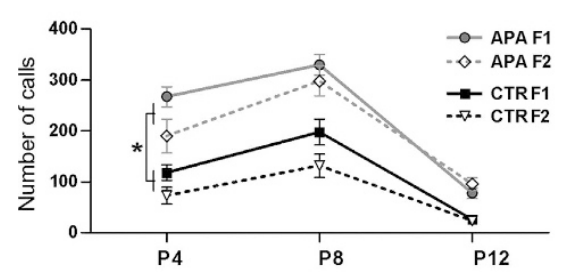

USV intensity

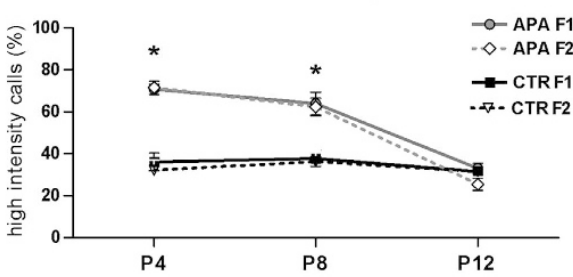

USV rate (nest material)

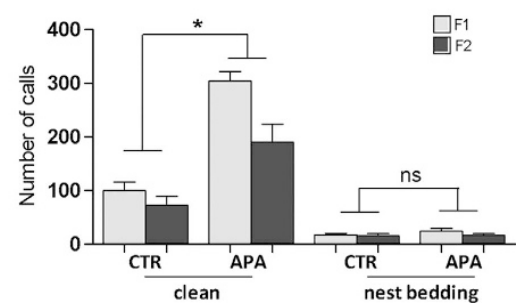

Figure 1. Increased USV activity in APA pups upon isolation from their mothers ( $n=25$ pups per generation per group). (a) Both generations of APA pups emitted an increased number of calls with respect to controls throughout postnatal development $(* P<0.001)$. (b) The percentage of high intensity calls was greater in F1 and F2 progenies derived from old fathers versus controls, on P4 and P8 $\left({ }^{*} P<0.001\right)$. (c) Although APA pups emitted more calls when isolated in clean Petri dishes $\left({ }^{*} P<0.001\right)$, no significant difference between the groups was observed when pups were isolated in the presence of nest materials (NS: $P=0.89$ ). APA, advanced paternal age; CTR, control; NS, not significant; USV, ultrasound vocalization. 
a

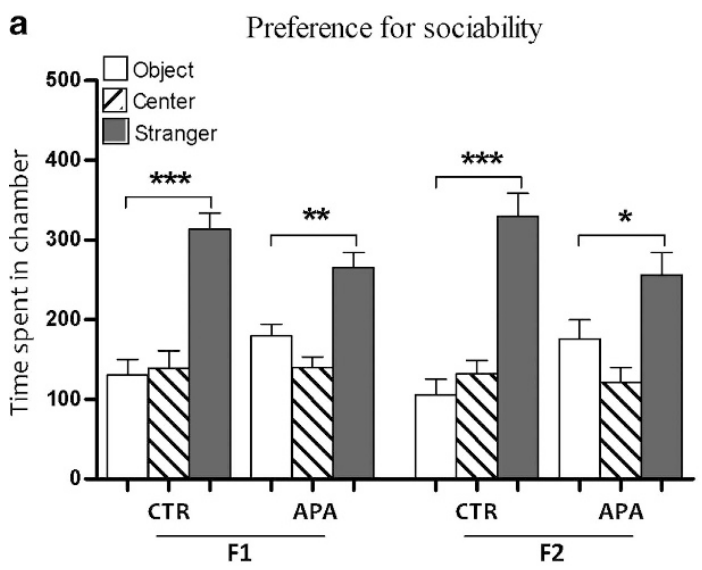

b

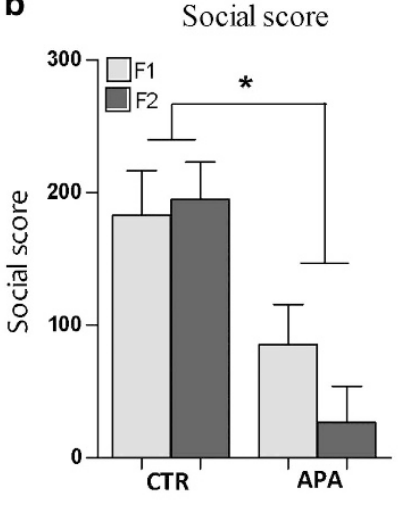

C

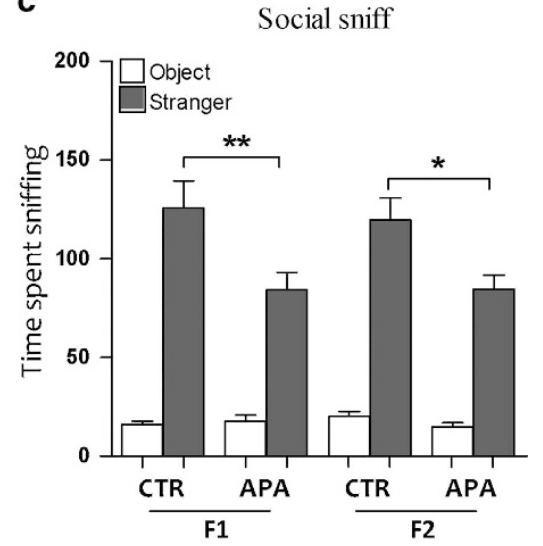

d Preference for social novelty

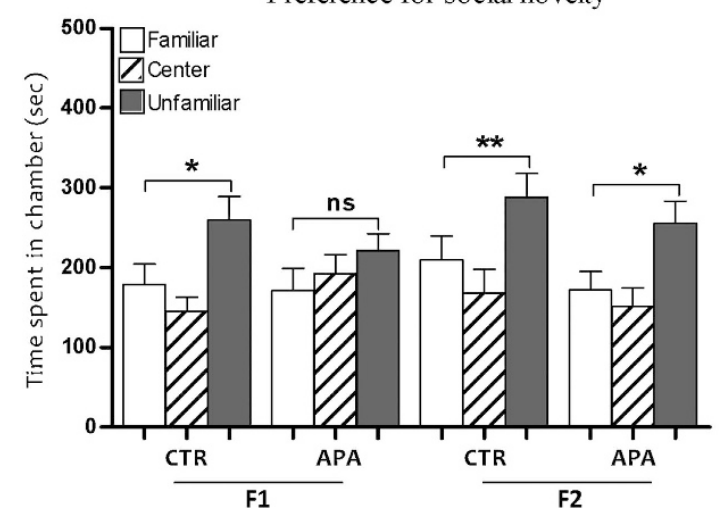

e

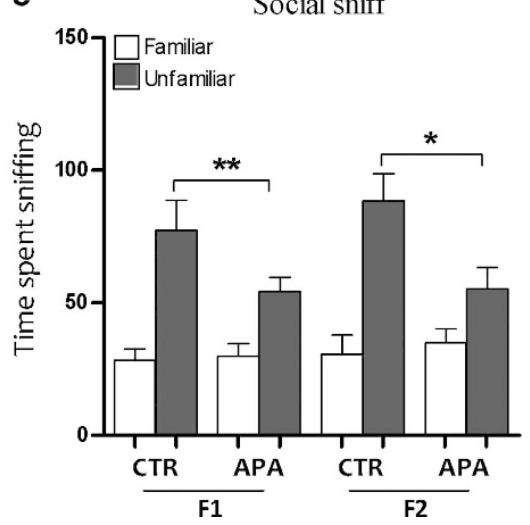

Figure 2. APA induced perturbations of social behaviors ( $n=15$ mice per generation per group). (a) All mice showed significant preferences for spending time in the chamber containing the stranger mouse $\left({ }^{*} P<0.05,{ }^{* *} P<0.01,{ }^{* * *} P<0.001\right)$, although the magnitude of preference was lower in APA progenies. (b) The sociability score was significantly lower in both generations of APA mice with respect to controls $(* P<0.05)$. (c) APA mice spent less time in social sniffing $\left({ }^{*} P<0.05\right.$, $\left.{ }^{* *} P<0.01\right)$. (d) In the test for social novelty, both generations of mice derived from young fathers spent significantly more time in the chamber containing the unfamiliar mouse $(* P<0.05$, $* * P<0.01)$. Conversely, APA F1 failed to display such a preference $\left({ }^{*} P<0.05\right.$, NS: $\left.P=0.16\right)$. (e) The time spent sniffing the wire cage containing the unfamiliar mouse over the familiar one, was significantly less in APA progenies versus the controls $\left({ }^{*} P<0.05,{ }^{* *} P<0.01\right)$. APA, advanced paternal age; CTR, control; NS, not significant.

Repetitive behaviors

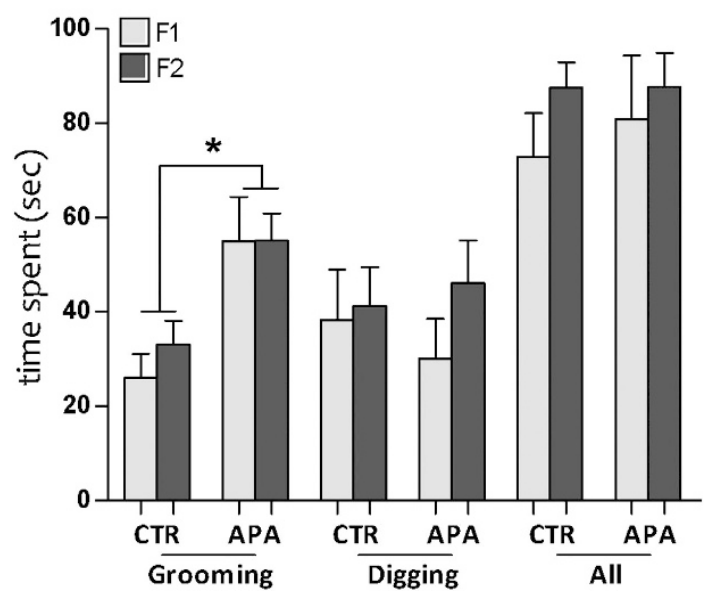

Figure 3. Increased self-grooming activity $(n=15$ mice per generation per group). APA progenies spent significantly more time in selfgrooming compared with controls $\left({ }^{*} P<0.05\right)$. Conversely, there were no significant differences in digging nor in the sum of all stereotyped behaviors analyzed. APA, advanced paternal age; CTR, control.

\section{Social deficits in APA progeny}

During the initial habituation trial of the sociability test, there were no preferences for empty chambers (side chamber 1 versus side chamber 2) across either APA and CTR mice or across generations. All mice analyzed showed a strong preference for the chamber housing a novel stranger mouse versus the chamber containing the novel object (Figure 2a). However, the magnitude of preference was decreased in APA F1 and F2 progeny, which showed a significant decrease in the sociability score compared with controls (Figure 2b). Although the time spent in social sniffing versus sniffing of the object was higher in all mice tested, APA progeny spent significantly less time in social sniffing compared with controls (Figure 2c). In the test for social novelty, CTR mice spent significantly more time in the chamber containing the unfamiliar mice. Conversely, APA mice failed to display a preference for the chamber containing the unfamiliar mouse over the chamber with the familiar mouse (Figure 2d). Moreover, the time spent sniffing the unfamiliar mouse was significantly less in APA mice compared with control mice (Figure 2e).

\section{Elevated levels of self-grooming in APA progeny}

The time spent grooming during the isolation period was significantly higher in APA F1 and F2 progeny compared with their respective controls. However, there were no significant 

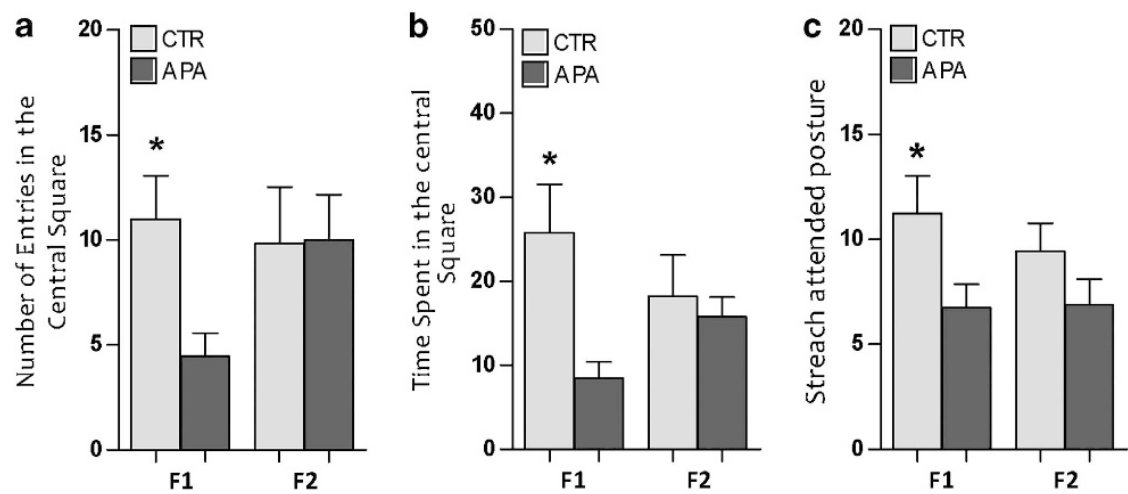

Figure 4. Effects of APA on anxiety-like behaviors ( $n=12$ mice per generation per group). (a, b) F1 mice derived from aged fathers displayed less number of entries into the central area of the open field with respect to the controls. Moreover the time spent in the central area was lower in APA with respect to the controls $(* P<0.05)$. (c) These mice also made significantly fewer stretch-attend postures in the open arms of the elevated plus maze than did the controls $\left({ }^{*} P<0.05\right)$. F2 progeny did not display changes in anxiety-like behaviors. APA, advanced paternal age; CTR, control.

differences in the time spent digging and in the sum of all stereotypic behaviors (Figure 3).

\section{Increased anxiety-like response in APA F1 offspring}

There was a significant effect of APA on anxiety-related behaviors. The number of entries into and the time spent in the central area of the open field were significantly less in APA F1 offspring than in controls (Figures $4 a$ and b). In the elevated plus maze, there were no differences between the groups in the number of entries into the open arms, nor in the time spent in them. However, APA mice made significantly fewer stretch-attend postures in the open arms than did controls (Figure 4c), suggesting an effect of APA on explorative behaviors. We did not find any significant differences in anxiety-related behaviors in F2 mice. Also locomotor activity was not different between APA and CTR, in both generations of mice analyzed.

\section{DISCUSSION}

We report that the mice conceived by aged fathers and their progeny exhibit autistic-like phenotypes that markedly resemble core symptoms of ASD. Both generations of mice-F1 derived from 15-month-old fathers, and F2 derived from 4-month-old parents and 15-month-old grandfather-displayed communication impairments, increased repetitive behavior and social deficits, whereas changes in anxiety-related behaviors were only significant in the first offspring generation. Social deficits, communication impairments and repetitive behaviors are considered diagnostic features of ASD, therefore our model specifically and selectively mimicked various core symptoms of ASD in humans. Moreover, this is the first and only model of a risk factor for autism in which transgenerational effects have been analyzed.

Communication between APA pups and their mothers was altered, as demonstrated by the increased USV activity during social isolation. Similar findings were reported in several mouse strains suggested to be relevant to autism studies. ${ }^{24,31-33}$ In particular, pups of the BTBR inbred mouse, which display many ASD-like symptoms, exhibit an increased number of USV calls. ${ }^{19}$ Alterations in USV parameters were also reported in engineered mice carrying deletions of genes associated with autism, ${ }^{17,20,21}$ and in rats as a model for ASD. ${ }^{41}$ The increased vocalization could be due to increased susceptibility to stress. USV emissions were increased only when APA offspring were isolated in clean Petri dishes; conversely, emissions were dramatically attenuated by the presence of nest materials, indicating that the presence of familiar social cues (that is, odor stimuli) is a support to reduce the stress response in isolated pups. In addition to communicative impairments, APA mice exhibited deficits in social behavior, as demonstrated by the decreased exploratory preference for the chamber containing a stranger mouse over the chamber containing a non-social novel object. Moreover, APA mice exhibited abnormal social behavior when choosing between an alreadyinvestigated mouse and a newly-introduced one. Although control mice spent significantly more time interacting with the unfamiliar mouse than the familiar one, APA mice did not display such a preference. Pro-social behaviors in mice include a general preference for social proximity to a stranger mouse versus a non-social novel object, a more specific preference for unfamiliar mice, and the ability to discriminate between a newly-introduced versus an already-investigated stranger. ${ }^{42}$ Overall, these behaviors were perturbed in the APA mice. These phenotypes may be analogous to the impairment in appropriate social interaction seen in autistic patients. ${ }^{43}$ Moreover, social abnormalities were also observed under similar experimental conditions, in mice with an autistic genetic background or those exposed to environmental risk factors. ${ }^{17,20,22,44,45}$

Furthermore, we observed elevated self-grooming behaviors in F1 and F2 APA mice, which spent significantly more time in whole body grooming during isolation in a novel cage environment. Selfgrooming in APA mice may represent the core symptom of increased repetitive behavior typical of ASD. A similar phenotype was observed in Neuroligin-1 and Neurexin-1a (candidate genes for autism) KO mice, which also displayed excessive grooming behavior. ${ }^{46,47}$ These genes are involved in a synaptic pathway in which dysfunctions of any of the genes involved can result in an autistic behavioral phenotype. ${ }^{25,48}$ Similar increase in repetitive behaviors, including excessive motor stereotypy (that is, digging and jumping behaviors), was observed in other ASD-model mice. $24,25,49$

Anxiety-related responses were altered in the first generation derived from the aged fathers, as APA mice spent less time exploring open spaces in both the open field and the elevated plus maze tests. Although anxiety is not essential for an ASD diagnosis, an estimated $40 \%$ of children with ASD fulfill diagnostic criteria for an anxiety disorder, ${ }^{50}$ and as many as $84 \%$ have subclinical anxiety symptoms. ${ }^{51}$ Despite the prevalence of anxiety in ASD, it remains unclear whether anxiety constitutes a separate condition with comorbidity with ASD or whether it aligns more closely with ASD's core features. ${ }^{52}$

We found that F2 mice derived from old grandfathers presented all three behavioral features of ASD (communication impairments, increased repetitive behavior and social deficits), despite being conceived by young fathers. Recent epidemiological studies in 
humans reported that the grandfather's age is associated with an increased risk of autism and schizophrenia, independent of paternal or maternal age. ${ }^{53,54}$ Interestingly, genome-wide sequencing studies indicate that men transmit a much higher number of mutations to their children than women, and that it is the age of the father that is the dominant factor in determining the mutation rate. ${ }^{14,16}$ Considering the association between autism and de novo mutations inherited by the parents, ${ }^{15,55,56}$ it is possible that agerelated mutability of genes expressed in the brain and implicated in the development of autism, could lead to heritable phenotypic changes in subsequent generations. Another possible explanation concerns the epigenetic integrity of germ cells. Dysregulation of epigenetic processes during spermatogenesis in older men can also contribute to the association between APA and ASD. Hypermethylation of ribosomal DNA and changes in chromatin packaging and integrity have been observed in the sperm of older rats. ${ }^{57,58}$ Moreover, a recent study reported that APA is associated with altered DNA methylation at brain-expressed imprinted loci in inbred mice. ${ }^{59}$ In addition, age-related DNA methylation changes have been widely reported, with individual sperm cells demonstrating significant intra- and interindividual epigenetic variation as a function of increasing male age. ${ }^{60,61}$ Overall, age-related factors can potentially alter genomic information carried by the sperm, either through epigenetic reprogramming or increased rates of de novo mutations, leading to the development of abnormal phenotypes in experimental animals and an increased disease predisposition in humans.

However, results reported here only describe the behavioral phenotype of the model, and further studies are needed to verify the presence of molecular and neurological biomarkers either in the brain and/or in the periphery of APA mice. Moreover, model ASD in mice still present some translational limitations, due to specie-specific differences in neurodevelopmental trajectories and specific behavioral outcome, which may be impossible to reproduce in mice (that is, theory of mind, speech language).

In conclusion, our study suggests that the risk of ASD could develop over generations, consistent with heritable mutations and/or epigenetic alterations associated with APA. On the basis of spontaneous age-related mutagenesis, this model represents a naturalistic platform, which might provide information about upstream mechanisms of action in autism, how mutations and epigenetic errors contribute to the development of ASD, as well as its neurobiology and pathogenesis, and could serve as translational platforms for development of treatments and for early diagnosis.

\section{CONFLICT OF INTEREST}

The authors declare no conflict of interest.

\section{ACKNOWLEDGMENTS}

This work was supported by the European Research Council (FP7/2007-2013)/ Programme IDEAS GA no. 210103 to GEP; Programme FP7-KBBE-2012.1.3-04, GA no. 312097 Acronym: FECUND, to GEP; MIUR/CNR, Programme FIRB. GA n. B81J12002520001 Acronym: GenHome, to PL. This study was also partially financed by the IGAB PAS project (S.III.1.3). The authors are participating in the COST action FA 1201 'Epiconcept' Epigenetic and Periconception Environment.

\section{REFERENCES}

1 Volkmar FR, Pauls D. Autism. Lancet 2003; 362: 1133-1141.

2 Abrahams BS, Geschwind DH. Advances in autism genetics: on the threshold of a new neurobiology. Nat Rev Genet 2008; 9: 341-355.

3 Geschwind DH. Autism: many genes, common pathways? Cell 2008; 135: 391-395.

4 Ramocki MB, Zoghbi HY. Failure of neuronal homeostasis results in common neuropsychiatric phenotypes. Nature 2008; 455: 912-918.
5 Hallmayer J, Cleveland S, Torres A, Phillips J, Cohen B, Torigoe T et al. Genetic heritability and shared environmental factors among twin pairs with autism. Arch Gen Psychiatry 2011; 68: 1095-1102.

6 Brown AS. Epidemiologic studies of exposure to prenatal infection and risk of schizophrenia and autism. Dev Neurobiol 2012; 72: 1272-1276.

7 Hultman CM, Sandin S, Levine SZ, Lichtenstein P, Reichenberg A. Advancing paternal age and risk of autism: new evidence from a population-based study and a meta-analysis of epidemiological studies. Mol Psychiatry 2011; 16: 1203-1212.

8 Reichenberg A, Gross R, Weiser M, Bresnahan M, Silverman J, Harlap S et al. Advancing paternal age and autism. Arch Gen Psychiatry 2006; 63: 1026-1032.

9 Tsuchiya KJ, Matsumoto K, Miyachi T, Tsujii M, Nakamura K, Takagai S et al. Paternal age at birth and high-functioning autistic-spectrum disorder in offspring. Br J Psychiatry 2008; 193: 316-321.

10 Durkin MS, Maenner MJ, Newschaffer CJ, Lee LC, Cunniff CM, Daniels JL et al. Advanced parental age and the risk of autism spectrum disorder. Am J Epidemiol 2008; 168: 1268-1276.

11 van Balkom ID, Bresnahan M, Vuijk PJ, Hubert J, Susser E, Hoek HW. Paternal age and risk of autism in an ethnically diverse, non-industrialized setting: Aruba. PloS One 2012; 7: e45090.

12 Perrin MC, Brown AS, Malaspina D. Aberrant epigenetic regulation could explain the relationship of paternal age to schizophrenia. Schizophr Bull 2007; 33: $1270-1273$

13 Crow JF. The origins, patterns and implications of human spontaneous mutation. Nat Rev Genet 2000; 1: 40-47.

14 Kong A, Frigge ML, Masson G, Besenbacher S, Sulem P, Magnusson G et al. Rate of de novo mutations and the importance of father's age to disease risk. Nature 2012; 488: 471-475.

15 Michaelson JJ, Shi Y, Gujral M, Zheng H, Malhotra D, Jin X et al. Whole-genome sequencing in autism identifies hot spots for de novo germline mutation. Cell 2012; 151: 1431-1442.

16 O'Roak BJ, Vives L, Girirajan S, Karakoc E, Krumm N, Coe BP et al. Sporadic autism exomes reveal a highly interconnected protein network of de novo mutations. Nature 2012; 485: 246-250.

17 Jamain S, Radyushkin K, Hammerschmidt K, Granon S, Boretius S, Varoqueaux F et al. Reduced social interaction and ultrasonic communication in a mouse model of monogenic heritable autism. Proc Natl Acad Sci USA 2008; 105: 1710-1715.

18 Moy SS, Nonneman RJ, Young NB, Demyanenko GP, Maness PF. Impaired sociability and cognitive function in Nrcam-null mice. Behav Brain Res 2009; 205: 123-131.

19 Scattoni ML, Gandhy SU, Ricceri L, Crawley JN. Unusual repertoire of vocalizations in the BTBR T+tf/J mouse model of autism. PloS One 2008; 3: e3067.

20 Gkogkas CG, Khoutorsky A, Ran I, Rampakakis E, Nevarko T, Weatherill DB et al. Autism-related deficits via dysregulated elF4E-dependent translational control. Nature 2013; 493: 371-377.

21 Nakatani J, Tamada K, Hatanaka F, Ise S, Ohta H, Inoue K et al. Abnormal behavior in a chromosome-engineered mouse model for human 15q11-13 duplication seen in autism. Cell 2009; 137: 1235-1246.

22 Malkova NV, Yu CZ, Hsiao EY, Moore MJ, Patterson PH. Maternal immune activation yields offspring displaying mouse versions of the three core symptoms of autism. Brain Behav Immun 2012; 26: 607-616.

23 Peca J, Feliciano C, Ting JT, Wang W, Wells MF, Venkatraman TN et al. Shank3 mutant mice display autistic-like behaviours and striatal dysfunction. Nature 2011; 472: 437-442.

24 Tsai PT, Hull C, Chu Y, Greene-Colozzi E, Sadowski AR, Leech JM et al. Autistic-like behaviour and cerebellar dysfunction in Purkinje cell Tsc1 mutant mice. Nature 2012; 488: 647-651.

25 Greco B, Kao HT, Valtorta F, Benfenati F. Autism-related behavioral abnormalities in synapsin knockout mice. Behav Brain Res 2012; 251: 65-74.

26 Garcia-Palomares S, Pertusa JF, Miñarro J, García-Pérez MA, Hermenegildo C, Rausell $\mathrm{F}$ et al. Long-term effects of delayed fatherhood in mice on postnatal development and behavioral traits of offspring. Biol Reprod 2009; 80: 337-342.

27 Smith RG, Kember RL, Mill J, Fernandes C, Schalkwyk LC, Buxbaum JD et al. Advancing paternal age is associated with deficits in social and exploratory behaviors in the offspring: a mouse model. PloS One 2009; 4: e8456.

28 Foldi CJ, Eyles DW, McGrath JJ, Burne TH. Advanced paternal age is associated with alterations in discrete behavioural domains and cortical neuroanatomy of C57BL/6J mice. Eur J Neurosci 2010; 31: 556-564.

29 Constantino JN, Todd RD. Autistic traits in the general population: a twin study. Arch Gen Psychiatry 2003; 60: 524-530.

30 Ricceri L, Markina N, Valanzano A, Fortuna S, Cometa MF, Meneguz A et al. Developmental exposure to chlorpyrifos alters reactivity to environmental and social cues in adolescent mice. Toxicol Appl Pharmacol 2003; 191: 189-201.

31 Hofer MA, Shair HN, Brunelli SA. Ultrasonic vocalizations in rat and mouse pups. Curr Protoc Neurosci 2002; Chapter 8: Unit 8.14. 
32 Oswalt GL, Meier GW. Olfactory, thermal, and tactual influences on infantile ultrasonic vocalization in rats. Dev Psychobiol 1975; 8: 129-135.

33 Lyons DM, Banks EM. Ultrasounds in neonatal rats: novel, predator and conspecific odor cues. Dev Psychobiol 1982; 15: 455-460.

34 Nadler JJ, Moy SS, Dold G, Trang D, Simmons N, Perez A et al. Automated apparatus for quantitation of social approach behaviors in mice. Genes Brain Behav 2004; 3: 303-314.

35 Yang M, Silverman JL, Crawley JN. Automated three-chambered social approach task for mice. Curr Protoc Neurosci 2011; Chapter 8: Unit 8.26.

36 Swiergiel AH, Dunn AJ. Effects of interleukin-1beta and lipopolysaccharide on behavior of mice in the elevated plus-maze and open field tests. Pharmacol Biochem Behav 2007; 86: 651-659.

37 Dunn AJ, Swiergiel AH. Behavioral responses to stress are intact in CRFdeficient mice. Brain Res 1999; 845: 14-20.

38 Juszczak GR, Sliwa AT, Wolak P, Tymosiak-Zielinska A, Lisowski P, Swiergiel AH. The usage of video analysis system for detection of immobility in the tail suspension test in mice. Pharmacol Biochem Behav 2006; 85: 332-338.

39 Swiergiel AH, Leskov IL, Dunn AJ. Effects of chronic and acute stressors and CRF on depression-like behavior in mice. Behav Brain Res 2008; 186: 32-40.

40 Haave M, Bernhard A, Jellestad FK, Heegaard E, Brattelid T, Lundebye AK. Longterm effects of environmentally relevant doses of 2,2',4,4',5,5' hexachlorobiphenyl (PCB153) on neurobehavioural development, health and spontaneous behaviour in maternally exposed mice. Behav Brain Funct 2011; 7: 3.

41 Burgdorf J, Moskal JR, Brudzynski SM, Panksepp J. Rats selectively bred for low levels of play-induced $50 \mathrm{kHz}$ vocalizations as a model for autism spectrum disorders: a role for NMDA receptors. Behav Brain Res 2013; 251: 18-24.

42 Ricceri L, Moles A, Crawley J. Behavioral phenotyping of mouse models of neurodevelopmental disorders: relevant social behavior patterns across the life span. Behav Brain Res 2007; 176: 40-52.

43 Wing L. The autistic spectrum. Lancet 1997; 350: 1761-1766.

44 Moy SS, Nadler JJ, Young NB, Perez A, Holloway LP, Barbaro RP et al. Mouse behavioral tasks relevant to autism: phenotypes of 10 inbred strains. Behav Brain Res 2007; 176: 4-20.

45 Zhang Y, Gao D, Kluetzman K, Mendoza A, Bolivar VJ, Reilly A et al. The maternal autoimmune environment affects the social behavior of offspring. I Neuroimmunol 2013; 258: 51-60.

46 Blundell J, Blaiss CA, Etherton MR, Espinosa F, Tabuchi K, Walz C et al. Neuroligin-1 deletion results in impaired spatial memory and increased repetitive behavior. $J$ Neurosci 2010; 30: 2115-2129.

47 Etherton MR, Blaiss CA, Powell CM, Sudhof TC. Mouse neurexin-1alpha deletion causes correlated electrophysiological and behavioral changes consistent with cognitive impairments. Proc Natl Acad Sci USA 2009; 106: 17998-18003.

48 Chih B, Gollan L, Scheiffele P. Alternative splicing controls selective trans-synaptic interactions of the neuroligin-neurexin complex. Neuron 2006; 51: 171-178.

49 Scattoni ML, Martire A., Cartocci G., Ferrante A., Ricceri L. Reduced social interaction, behavioural flexibility and BDNF signalling in the BTBR $T+t f / J$ strain, a mouse model of autism. Behav Brain Res 2012; 251: 35-40.
50 van Steensel FJ, Bogels SM, Perrin S. Anxiety disorders in children and adolescents with autistic spectrum disorders: a meta-analysis. Clin Child Fam Psychology Rev 2011; 14: 302-317.

51 White SW, Oswald D, Ollendick T, Scahill L. Anxiety in children and adolescents with autism spectrum disorders. Clin Psychol Rev 2009; 29: 216-229.

52 Kerns CM, Kendall PC. The presentation and classification of anxiety in autism spectrum disorder. Clin Psychol 2013; 19: 323-347.

53 Frans EM, Sandin S, Reichenberg A, Långström N, Lichtenstein P, McGrath JJ et al. Autism risk across generations: a population-based study of advancing grandpaternal and paternal age. JAMA Psychiatry 2013; 70: 516-521.

54 Frans EM, McGrath JJ, Sandin S, Lichtenstein P, Reichenberg A, Långström N et al. Advanced paternal and grandpaternal age and schizophrenia: a three-generation perspective. Schizophr Res 2011; 133: 120-124.

55 O'Roak BJ, Deriziotis P, Lee C, Vives L, Schwartz J, Girirajan S et al. Exome sequencing in sporadic autism spectrum disorders identifies severe de novo mutations. Nat Genet 2011; 43: 585-589.

56 Sanders SJ, Murtha MT, Gupta AR, Murdoch JD, Raubeson MJ, Willsey AJ et al. De novo mutations revealed by whole-exome sequencing are strongly associated with autism. Nature 2012; 485: 237-241.

57 Zubkova EV, Robaire B. Effects of ageing on spermatozoal chromatin and its sensitivity to in vivo and in vitro oxidative challenge in the Brown Norway rat. Hum Reprod 2006; 21: 2901-2910.

58 Oakes CC, Smiraglia DJ, Plass C, Trasler JM, Robaire B. Aging results in hypermethylation of ribosomal DNA in sperm and liver of male rats. Proc Natl Acad Sci USA 2003; 100: 1775-1780.

59 Smith RG, Reichenberg A, Kember RL, Buxbaum JD, Schalkwyk LC, Fernandes C et al. Advanced paternal age is associated with altered DNA methylation at brainexpressed imprinted loci in inbred mice: implications for neuropsychiatric disease. Mol Psychiatry 2013; 18: 635-636.

60 Hernandez DG, Nalls MA, Gibbs JR, Arepalli S, van der Brug M, Chong S et al. Distinct DNA methylation changes highly correlated with chronological age in the human brain. Hum Mol Genet 2011; 20: 1164-1172.

61 Flanagan JM, Popendikyte V, Pozdniakovaite N, Sobolev M, Assadzadeh A, Schumacher $A$ et al. Intra- and interindividual epigenetic variation in human germ cells. Am J Hum Genet 2006; 79: 67-84.

(c) (i) $\Theta$ This work is licensed under a Creative Commons Attributioncc. NonCommercial-NoDerivs 3.0 Unported License. The images or other third party material in this article are included in the article's Creative Commons license, unless indicated otherwise in the credit line; if the material is not included under the Creative Commons license, users will need to obtain permission from the license holder to reproduce the material. To view a copy of this license, visit http://creativecommons.org/licenses/by-nc-nd/3.0/

Supplementary Information accompanies the paper on the Translational Psychiatry website (http://www.nature.com/tp) 